\title{
UNION AND TANGLE
}

\author{
YASUTAKA NAKANISHI
}

(Communicated by Ronald J. Stern)

\begin{abstract}
Shibuya proved that any union of two nontrivial knots without local knots is a prime knot. In this note, we prove it in a general setting. As an application, for any nontrivial knot, we give a knot diagram such that a single unknotting operation on the diagram cannot yield a diagram of a trivial knot.
\end{abstract}

\section{INTRODUCTION}

In 1957, Kinoshita and Terasaka [8] introduced the notion of union of knots, which is a generalization of a connected sum as indicated in Figure 1. For the connected sum of two knots, $k_{1} \# k_{2}$, we consider an arc $\alpha$ which intersects a decomposing sphere in a single point such that the arc $\alpha$ meets $k_{1} \# k_{2}$ in its endpoints, one endpoint is on $k_{1}$ and another endpoint is on $k_{2}$. By winding the subarcs of $k_{1}$ and $k_{2}$ along the arc $\alpha$, we obtain a new knot or link, which is called a union of $k_{1}$ and $k_{2}$. If there are no local knots in the exterior of the decomposing sphere and the arc $\alpha$, it is called a union without local knots. For example, a union of two prime knots is a union without local knots, if the arc cannot be isotoped to miss the decomposing sphere. In a sense, any knot can be obtained from trivial knots by a finite sequence of union. In fact, the given knot $k$ is obtained by the union of a trivial knot and a certain knot $k^{\prime}$ with $u\left(k^{\prime}\right)=u(k)-1$.

In 1993, Shibuya [15] proved that any union of two nontrivial knots without local knots is a prime knot. In this note, we prove it in a general setting.
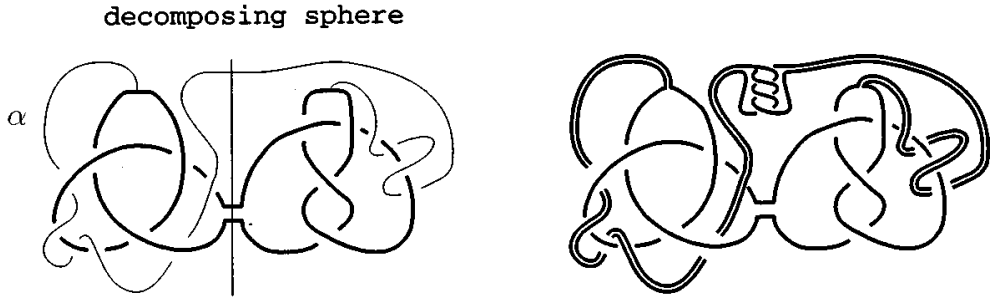

FiguRe 1

Received by the editors November 3, 1994.

1991 Mathematics Subject Classification. Primary 57M25.

Key words and phrases. Union, tangle, primeness, Montesinos knot. 


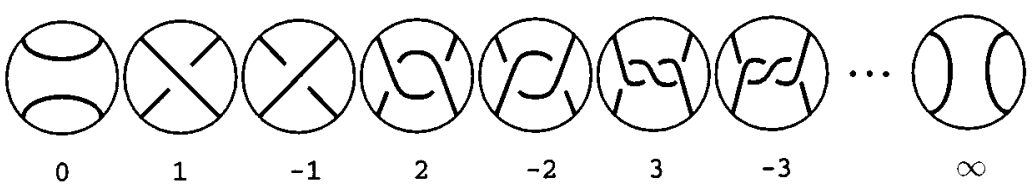

Figure 2

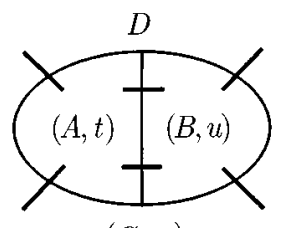

$(C, v)$

FiguRE 3

\section{TANGLES}

In this note, a tangle means a 2 -string tangle, which is a pair $(B, t)$ of a 3 -ball $B$ and two arcs $t$ properly embedded in $B$. If a tangle has no local knots, the tangle is said to be locally trivial. Two tangles are said to be equivalent if there is a homeomorphism of pairs from one tangle to another. If two arcs in a tangle can be separated by a 2-disc properly embedded in a 3-ball, the tangle is said to be separable. If a tangle is locally trivial and separable, the tangle is said to be trivial. A regular diagram of a trivial tangle, or its spatial realization, is said to be rational. In a special class of rational tangles, there are integral tangles as in Figure 2, where two arcs are winding by horizontally $n$ (right) half-twists. They are sometimes called by the number of half-twists, $n$. In the case of $\infty$, the tangle has two arcs vertically parallel. If a tangle is locally trivial and nonseparable, the tangle is said to be prime.

The following Propositions 1 and 2 are known by Lickorish [9].

Proposition 1. Let $k$ be a knot or link in the 3 -sphere $S^{3}$. Suppose that $S^{2}$ is a 2-sphere in $S^{3}$ meeting $k$ transversely in four points and separating $S^{3}$ into two 3-balls $A$ and $B$. If both tangles $(A, A \cap k)$ and $(B, B \cap k)$ are prime, then $k$ is prime.

Proposition 2. Let $(C, v)$ be a tangle and $D$ a disc properly embedded in $C$ such that $D$ separates $(C, v)$ into two tangles $(A, t)$ and $(B, u)$ and that the numbers of points of $(\partial A \backslash D) \cap v,(\partial B \backslash D) \cap v, D \cap v$ are all two. Suppose that $(B, u)$ is a prime tangle and $\partial D$ is not contractible in $A \backslash t$. Then the tangle $(C, v)$ is a prime tangle.

Proposition 3. Let $(C, v)$ be a tangle and $D$ a disc properly embedded in $C$ such that $D$ separates $(C, v)$ into two tangles $(A, t)$ and $(B, u)$ as indicated in Figure 3, and that the numbers of points of $(\partial A \backslash D) \cap v,(\partial B \backslash D) \cap v, D \cap v$ are all two. Suppose that both tangles $(A, t)$ and $(B, u)$ are trivial and there is no homeomorphism of pairs from $(A, t)$ (and from $(B, u)$ ) in Figure 3 to an integral tangle with fixing boundaries. Then the tangle $(C, v)$ is a prime tangle. 


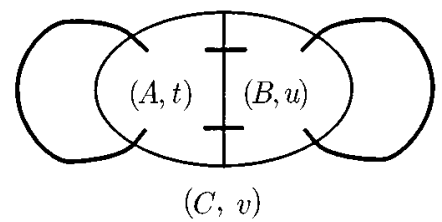

FiguRE 4

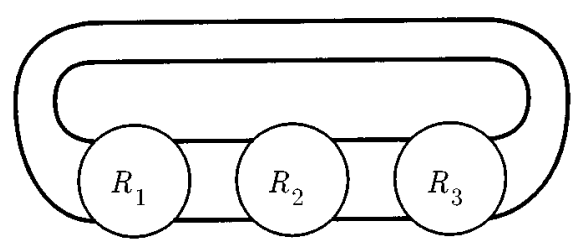

FIGURE 5

Proof. Consider the knot (or link) $k$ obtained from the tangle $(C, v)$ with ears as indicated in Figure 4. As both tangles $(A, t)$ and $(B, u)$ are rational and neither tangle $(A, t)$ nor $(B, u)$ is integral, the result $k$ is a 3 -bridge knot (i.e. the connected sum of two 2-bridge knots). If the tangle $(C, v)$ is trivial, $k$ might be a 2-bridge knot. It is a contradiction. The proof of local triviality of $(C, v)$ is easy and essentially contained in [9], and so we omit it here. The proof is complete.

\section{Montesinos KNOTS AND LINKS}

Montesinos [11, 12] defined a set of knots and links whose two-fold branched covering spaces are Seifert fibre spaces. In this note, we need only Montesinos knots and links with the three strands, which are indicated in Figure 5, where $R_{1}, R_{2}, R_{3}$ mean rational tangles.

Montesinos knots and links are classified by Bonahon [5] and by Boileau and Siebenmann [4]. As corollary of their results, we have the following.

Proposition 4. Suppose $k$ is a Montesinos knot (or link) with three strands as indicated in Figure 5. If none of the three rational tangles $R_{1}, R_{2}, R_{3}$ are integral, then $k$ is a prime 3 -bridge knot.

\section{Main Theorem}

Main Theorem. Let $k$ be a knot (or link) obtained from one rational tangle $R$ and two tangles $T_{1}$ and $T_{2}$, which are locally trivial tangles but are not integral tangles as indicated in Figure 6 . If $R$ is not integral, then $k$ is prime.

Proof. The proof is separated into the three cases as follows. (1) Both tangles $T_{1}$ and $T_{2}$ are prime. (2) One of the tangles $T_{1}$ and $T_{2}$ is prime and another is trivial. (3) Both tangles $T_{1}$ and $T_{2}$ are trivial.

(1) From Proposition 2, the tangle obtained by joining $T_{1}$ with $R$ is a prime tangle. Since the tangle $T_{2}$ is also prime, the result $k$ is prime by Proposition 1 .

(2) For convenience, let $T_{1}$ be trivial. As a hypothesis, the tangle $T_{1}$ is not integral. From Proposition 3, the tangle obtained by joining $T_{1}$ with $R$ is a prime tangle. Since the tangle $T_{2}$ is also prime, the result $k$ is prime by Proposition 1. 


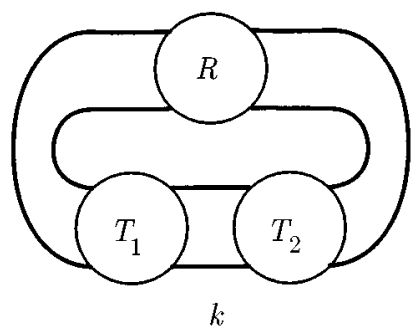

FiguRE 6

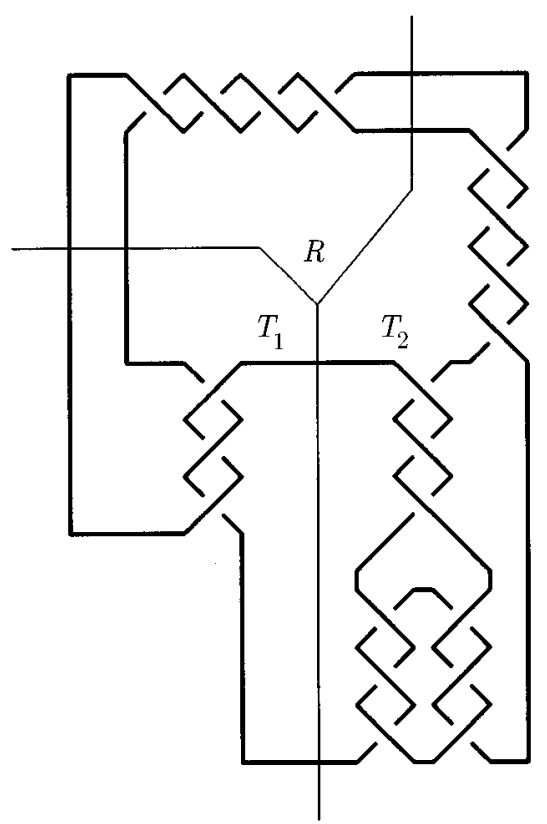

FiguRE 7

(3) As a hypothesis, neither tangle $T_{1}$ nor $T_{2}$ is integral. Therefore, the result $k$ is a Montesinos knot (or link) with three strands satisfying the condition in Proposition 4 . Hence $k$ is prime. The proof is complete.

Remark. In the statement of the Main Theorem, the reason to omit the case of integral tangles is from the existence of counterexamples as indicated in Figure 7.

As a corollary of the Main Theorem, we have the following result on a union of two nontrivial knots.

Corollary. Let $k_{1}$ and $k_{2}$ be nontrivial knots, which are presented by locally trivial tangles $T_{1}$ and $T_{2}$ with ears as indicated in the left of Figure 8. Suppose that $k$ is a knot (or link) obtained from two tangles $T_{1}$ and $T_{2}$ and one rational tangle $R$ as indicated in the right of Figure 8. If $R$ is not integral, then $k$ is prime.

Proof. Since the two knots $k_{1}$ and $k_{2}$ are nontrivial, the two tangles $T_{1}$ and $T_{2}$ are 


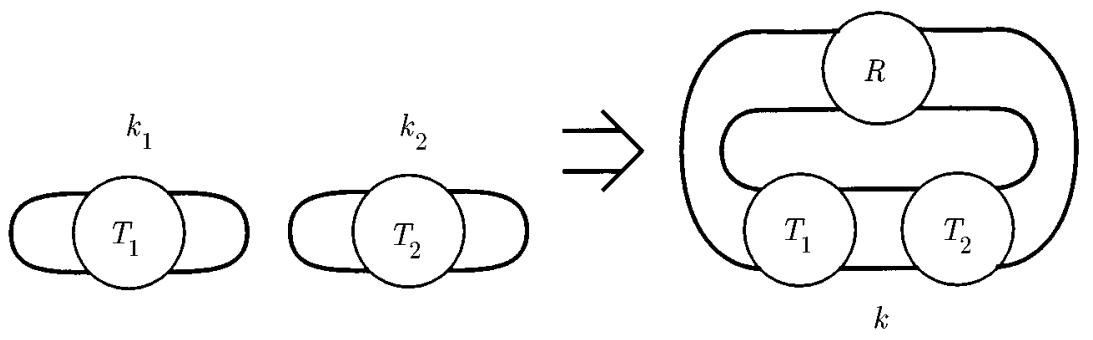

Figure 8

not integral. From the Main Theorem above, we have the required result. The proof is complete.

\section{INSIDE STORY OF TANGLE}

The development of knot theory in the 1980s gave us many results and tools. The solution of Property P for strongly-invertible knots, the solution of Property $\mathrm{R}$, and the super-additivity of genus on band-sum enable us to show the following facts easily. The proofs can be seen in several papers, for instance, [2], [3], [6], [14].

Proposition 5. For a nontrivial tangle, there is at most one attachment with a trivial tangle to yield a trivial knot.

Proposition 6. For a nontrivial tangle, there is at most one attachment with a trivial tangle to yield a trivial 2-component link.

Proposition 7. For a tangle, suppose that there are an attachment with a trivial tangle to yield a trivial knot and an attachment with a trivial tangle to yield a trivial 2-component link. Then the given tangle is trivial.

Related to these topics, there is a problem on tangles as follows.

Problem. For a tangle, how many attachments with a trivial tangle that yield composite knots and links exist?

Eudave-Muñoz [6] proved the number is at most three, but the example to establish it is still unknown. He also gave an example which has two attachments with a trivial tangle to yield composite knots (cf. [1]). This example is closely related to the example in Figure 7.

We can give an elementary proof for the following fact:

Proposition 8. Trivial knots, 2-bridge knots, and torus knots are unchanged by mutation.

Proof. Let $k$ be either a trivial knot, 2-bridge knot, or torus knot. Suppose that $\left(S^{3}, k\right)$ is decomposed into two tangles. Since $k$ is a prime knot, the two tangles are assumed to be locally trivial. (Otherwise, one of the two tangles must be trivial.) It is known that $k$ cannot be decomposed into two prime tangles (furthermore, not into two $n$-string prime tangles): For the case of a trivial knot or 2-bridge knot, the proof is given by Lickorish [9]. For the case of a torus knot, the proof was given by Kawauchi and the author as stated in [7]: If $k$ were decomposed into two prime tangles $(A, A \cap k)$ and $(B, B \cap k)$, then the complement $\Sigma \backslash k$ of $k$ in the 
decomposing sphere $\Sigma$ is incompressible in both sides. Therefore, the knot group $\pi\left(S^{3} \backslash k\right)$ of $k$ is the free product of the fundamental groups $\pi(A \backslash k)$ and $\pi(B \backslash k)$ of the complements of $k$ in both tangles, with the amalgamated subgroup $\pi(\Sigma \backslash k)$. If the knot group $\pi\left(S^{3} \backslash k\right)$ has a center, then the center must be contained in the amalgamated subgroup (see [10]). The amalgamated subgroup $\pi(\Sigma \backslash k)$ is a free group of rank three, and it has a trivial center. On the other hand, the knot group of a torus knot has a nontrivial center $\mathbf{Z}$. It is a contradiction. Therefore, one of the two tangles is a trivial tangle. A trivial tangle cannot be changed by mutation. Hence, $k$ is also unchanged by mutation. The proof is complete.

\section{UNKNOTTING NUMBERS AND KNOT DIAGRAMS}

In [13], the author gave a knot diagram of the unknotting number one knot $6_{2}$ such that a single unknotting operation on the knot diagram cannot yield a knot diagram of trivial knot. By the Main Theorem above, we can give a knot diagram for any nontrivial knot such that a single unknotting operation on the knot diagram cannot yield a knot diagram of trivial knot. It is clear for knots with unknotting number two or more. For an unknotting number one knot, we deform a knot diagram as follows.

For a knot diagram, there is at most a finite number of crossings where a single unknotting operation yields a knot diagram of trivial knot. At each such crossing, we deform a neighborhood of the crossing as in Figure 9. Except crossings marked with and a a single unknotting operation yields a knot diagram of nontrivial knot, since the resulting knot has the original knot as a factor with respect to connected-sum.

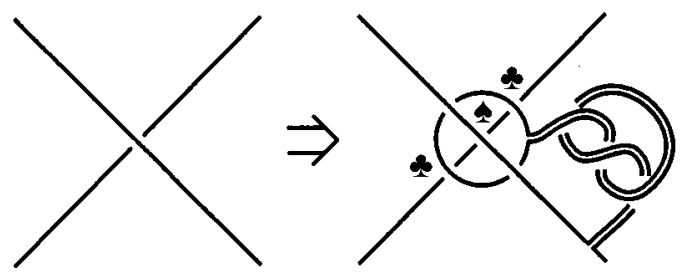

Figure 9

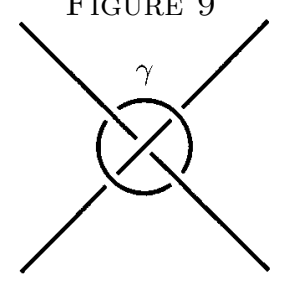

FiguRe 10

At the crossing marked with $\mathbf{a}$, a single unknotting operation yields a knot diagram of nontrivial knot. That is because the resulting knot satisfies the condition of the Main Theorem in the case of (1) or (2). In this case, the tangle corresponding to two parallel knotted arcs is a prime tangle. If the other tangle might have a local 
knot, the proof was clear. So, we can assume the other tangle has no local knot, and this situation satisfies the case (1) or (2) in the proof.

At the crossing marked with $\mathbf{\phi}$, a single unknotting operation yields a knot diagram of nontrivial knots, too, since the two parallel knotted arcs in the 3-ball is a prime tangle. As in the previous section 5, there is at most one attachment with a trivial tangle to yield a trivial knot. And any attachment with a nontrivial tangle yields a nontrivial knot. It is clearly seen that only one attachment of the two parallel knotted arcs in the 3-ball with a trivial tangle yields a trivial knot. In this case, the loop $\gamma$ as in Figure 10 must bound a disc in the complement of the knot, since the knot is geometrically essential in the solid torus $S^{3} \backslash{ }^{\circ} N(\gamma)$. This is a contradiction. Therefore, we can have the required result.

\section{ACKNOWLEDGEMENT}

The author would like to express his gratitude to the referee, who gave valuable suggestions and showed that the Corollary can be also proved applying the results of $[6]$.

\section{REFERENCES}

[1] S. A. Bleiler, Prime tangles and composite knots, Knot Theory and Manifolds, Lecture Notes in Math., vol. 1144, Springer-Verlag, Berlin and New York, 1985, pp. 1-13. MR 87e:57006

[2] S. A. Bleiler and M. Scharlemann, Tangles, property P, and a problem of J. Martin, Math. Ann. 273 (1986), 215-225. MR 87h:57007

[3] S. A. Bleiler and M. Scharlemann, A projective plane in $\mathbf{R}^{4}$ with three critical points are standard, Topology 27 (1988), 519-540. MR 90e:57006

[4] M. Boileau and L. Siebenmann, A planar classification of pretzel knots and Montesinos knots, Prépublications, Orsay, 1980.

[5] F. Bonahon, Involutions et fibrés de Seifert dans les variétés de dimension 3, Thèse de 3e cycle, Orsay, 1979.

[6] M. Eudave-Muñoz, Primeness and sums of tangles, Trans. Amer. Math. Soc. 306 (1988), 773-790. MR 89g:57005

[7] T. Kanenobu, Hyperbolic links with Brunnian properties, J. Math. Soc. Japan 38 (1986), 295-308. MR 88a:57024

[8] S. Kinoshita and H. Terasaka, On union of knots, Osaka Math. J. 9 (1957), 131-153. MR 20:4846

[9] W. B. R. Lickorish, Prime knots and tangles, Trans. Amer. Math. Soc. 267 (1981), 321-332. MR 83d:57004

[10] W. Magnus, A. Karrass, and D. Solitar, Combinatorial group theory: presentations of groups in terms of generators and relators, Interscience Publ., Wiley \& Sons, New York, 1966. MR 34:7617

[11] J. M. Montesinos, Una familia infinita de nudos representados no separables, Revista Math. Hisp.-Amer. (IV) 33 (1973), 32-35. MR 52:6698

[12] J. M. Montesinos, Revêtements ramifiés de nœuds, espaces fibrés de Seifert et scindements de Heegaard, Prépublications, Orsay, 1979.

[13] Y. Nakanishi, Unknotting numbers and knot diagrams with the minimum crossings, Math. Seminar Notes Kobe Univ. 11 (1983), 257-258. MR 85h:57008

[14] M. Scharlemann, Smooth spheres in $\mathbf{R}^{4}$ with four critical points are standard, Invent. Math. 79 (1985), 125-141. MR 86e:57010

[15] T. Shibuya, Primeness of union of knots, preprint (1993).

Department of Mathematics, Kobe University, 1-1, Rokkodai-Cho, Nada-Ku, Kobe 657, JAPAN

E-mail address: nakanisi@math.s.kobe-u.ac.jp 